\title{
The measurement of self-diffusion coefficients in liquid metals with quasielastic neutron scattering
}

\author{
Andreas Meyer ${ }^{\mathrm{a}}$ \\ Institut für Materialphysik im Weltraum, Deutsches Zentrum für Luft- und Raumfahrt (DLR), 51170 Köln, Germany
}

\begin{abstract}
Quasielastic incoherent neutron scattering (QENS) has proven to be a versatile tool to study self diffusion of atoms in liquid metals. Here it is shown, that coherent contributions to the signal in the small $q$ limit appear as a flat and energy independent constant to the QENS signal in single-component liquid metals even for systems with a small incoherent scattering cross section, like aluminum. Container-less processing via electromagnetic or electrostatic levitation devices, especially designed for QENS, enables the in-situ measurement on liquid metallic droplets of sizes between $5 \mathrm{~mm}$ to $10 \mathrm{~mm}$ in diameter. This gives access to the study of chemically reactive, refractory metallic melts and extends the accessible temperature range to undercoolings of several hundred Kelvin below the respective melting point. Compared to experiments using a thin-walled crucible giving hollow-cylindrical sample geometry it is shown that multiple scattering on levitated droplets is negligible for the analysis of the self-diffusion coefficient. QENS results of liquid germanium and ${ }^{73}$ germanium isotope mixtures, titanium, nickel, copper and aluminum are reviewed. The self-diffusion coefficients of these systems are best described by an Arrhenius-type temperature dependence around their respective melting points.
\end{abstract}

\section{Introduction}

Driven by density and concentration fluctuations, diffusion of mass is a fundamental property of liquids [1-3]. The understanding of atomic diffusion processes is a prerequisite for the study of liquid dynamics [4,5], nucleation and crystal growth [6-8], as well as vitrification $[9,10]$. Diffusion data serve as an input to the modeling of microstructure evolution $[11,12]$ and are an essential benchmark to molecular dynamics (MD) simulation results $[13,14]$. In general, self-diffusion coefficients are related to the mean square displacement of the tagged atoms, whereas interdiffusion coefficients are related to collective transport of mass driven by differences in the chemical potential, e.g. due to concentration gradients in alloys.

A common method to measure diffusion coefficients in liquid metals and alloys is the long capillary (LC) technique and its variations [15-18]. Usually a diffusion couple of different composition (in the case of interdiffusion), or containing a different amount of isotopes (in the case of self diffusion), is annealed in the liquid state and subsequently quenched to ambient temperature. The diffusion profiles are analyzed post mortem applying Fick's law of diffusion. However, in most cases the influences of convective flow on the evolving diffusion profile during annealing are not known. A comparison to long capillary experiments under microgravity conditions in space, where at least gravity driven convective flow is suppressed, shows that convective contributions indeed influence the measurement, the more so the larger the temperatures involved [16]. As a consequence, selfdiffusion coefficients are usually overestimated by several $10 \%$ to $100 \%$. Even their temperature dependence may

a e-mail: andreas.meyer@dlr.de exhibit systematic deviations from the actual one without convection [19]. As has been shown recently by in-situ monitoring of liquid interdiffusion processes with X-ray [20] or neutron radiography [21] on binary alloys with a sufficiently large difference in density of the diffusion couple, a stable density layering can suppress convective flow [22]. However, this does not apply to singlecomponent liquids. Therefore, accurate experimental selfdiffusion data in liquid metals are rare.

Self-diffusion coefficients in liquid metals can be measured in-situ with quasielastic neutron scattering [23,24]. QENS probes the dynamics on atomic length scales and on a picosecond time scale, short enough to be undisturbed by the presence of convective flow. In the case of an incoherent scattering contribution, the small- $q$ QENS signal is dominated by the incoherent contributions to the scattering signal. From the resulting intermediate scattering function the self-diffusion coefficient can be obtained on an absolute scale [19,25].

Recently we advanced the field of self-diffusion studies in metallic liquids by the use of container-less processing of liquid droplets. A combination of electromagnetic [26] or electrostatic levitation [27] of the samples with QENS enables the in-situ investigation of chemically reactive as well as of deeply undercooled metallic liquids.

In this article the technical aspects for the measurement of self-diffusion coefficients with quasielastic neutron scattering are reviewed and recent achievements in this field are summarized. In the example of liquid $\mathrm{Ge}$ and ${ }^{73} \mathrm{Ge}$ isotope mixtures, the scattering contributions to the signal are discussed and it is shown that the quasielastic signal at small wavenumber $q$ is dominated by incoherent scattering even for systems with a large ratio of coherent to incoherent scattering [28]. QENS results on liquid

This is an Open Access article distributed under the terms of the Creative Commons Attribution License 4.0, which permits unrestricted use, distribution, and reproduction in any medium, provided the original work is properly cited. 
titanium are shown in order to illustrate how accurate and precise self-diffusion coefficients can be derived from the QENS signal [13,29]. With two measurements on liquid nickel - one on a liquid droplet with $7 \mathrm{~mm}$ in diameter and the other one on a liquid sample in a thinwalled hollow-cylindrical $\mathrm{Al}_{2} \mathrm{O}_{3}$ sample holder, we show that the resulting QENS line width at half width at half maximum is not altered by multiple scattering effects $[26,30]$. Finally, it is shown that even for very weak incoherent scatterers like aluminum, self-diffusion coefficients can be derived at temperatures close to the melting point [31], and in combination with coherent QENS data [32], also their temperature dependence can be studied.

\section{QENS signal contributions}

Dynamic structure factors may be divided into a coherent and an incoherent part. The coherent dynamic structure factor reflects the collective motion of atoms, whereas the incoherent part reflects motions of single particles. In the hydrodynamic limit, i.e. in the limit of small wavenumbers $q$ and low frequencies $\omega$, the coherent dynamic structure factor in a liquid consists of a central Lorentzian shaped Rayleigh line, due to temperature fluctuations, and two Lorentzian shaped Brillouin lines, due to density fluctuations, dispersing with the velocity of sound, according to [33]:

$$
\begin{aligned}
2 \pi \frac{S(q, \omega)}{S(q)}= & \frac{\gamma-1}{\gamma}\left(\frac{2 D_{\mathrm{T}} q^{2}}{\omega^{2}+\left(D_{\mathrm{T}} q^{2}\right)^{2}}\right) \\
& +\frac{1}{\gamma}\left(\frac{\Gamma_{\mathrm{s}} q^{2}}{\left(\omega-c_{\mathrm{s}} q\right)^{2}+\left(\Gamma_{\mathrm{s}} q^{2}\right)^{2}}\right) \\
& +\frac{1}{\gamma}\left(\frac{\Gamma_{\mathrm{s}} q^{2}}{\left(\omega+c_{\mathrm{s}} q\right)^{2}+\left(\Gamma_{\mathrm{s}} q^{2}\right)^{2}}\right) .
\end{aligned}
$$

Here $\gamma=c_{\mathrm{P}} / c_{\mathrm{V}}$ denotes the ratio of specific heat at constant pressure and constant volume, $D_{\mathrm{T}}$ the thermal diffusivity, $c_{\mathrm{s}}$ the adiabatic velocity of sound and $\Gamma_{\mathrm{s}}$ a sound attenuation constant. In the hydrodynamic limit the incoherent dynamic structure factor consists of a single central Lorentzian curve according to [33]:

$$
S_{\text {inc }}(q, \omega)=\frac{1}{\pi} \frac{D q^{2}}{\omega^{2}+\left(D q^{2}\right)^{2}} .
$$

$D$ denotes the self-diffusion coefficient of the atoms.

In the following, the question of how incoherent quasielastic spectra are altered by coherent scattering contributions is addressed in the example of two different liquid Germanium samples, containing either ${ }^{\text {nat }} \mathrm{Ge}$ or a ${ }^{\text {nat } / 73} \mathrm{Ge}$ 50:50 isotope mixture. The incoherent neutron scattering cross section of the isotope mixture is approximately seven times larger than for ${ }^{\text {nat }} \mathrm{Ge}$. Dynamic structure factors are compared with coherent dynamic structure factors from X-ray scattering [34] and simulations [35]. It is shown that quasielastic broadening in the neutron $S(q, \omega)$ is dominated by incoherent scattering for wavenumbers of $0.3<q<0.8 \AA^{-1}$ and, thus, accurate self-diffusion coefficients can be measured on an absolute scale with QENS.

QENS studies of liquid germanium were performed on the neutron time-of-flight spectrometer ToFToF at the FRM II in Munich [36,37]. An incoming neutron wavelength of $\lambda=7 \AA$ was chosen, providing an accessible wavenumber range of $0.3 \AA^{-1}<q<1.6 \AA^{-1}$ with an energy resolution of $50 \mu \mathrm{eV}$ full width at half maximum. Samples were prepared from high purity ${ }^{\text {nat }} \mathrm{Ge}(99.999 \%)$ purchased from PPM pure metals $\mathrm{GmbH}$ and high purity ${ }^{73} \mathrm{Ge}$ isotope, with a $99.40 \%$ isotope enrichment and metal impurities in the ppm range, purchased from STB Isotope Germany $\mathrm{GmbH}$. Experiments were performed using either ${ }^{\text {nat }} \mathrm{Ge}$ or a 1:1 mixture of ${ }^{\text {nat }} \mathrm{Ge}$ and ${ }^{73} \mathrm{Ge}$. ${ }^{\text {nat }} \mathrm{Ge}$ yields a coherent neutron scattering cross section of $\sigma_{\mathrm{coh}}=8.42$ barn and an incoherent neutron scattering cross section of $\sigma_{\text {inc }}=$ 0.18 barn. The isotope mixture yields $\sigma_{\text {coh }}=5.46$ barn and $\sigma_{\text {inc }}=1.2$ barn. Moreover, the ratio of incoherent to coherent scattering cross section is an order of magnitude higher for the isotope mixture with 0.22 than for ${ }^{\text {nat }} \mathrm{Ge}$ with 0.02 .

Samples were processed in $\mathrm{Al}_{2} \mathrm{O}_{3}$ cylinders having a wall thickness of $0.5 \mathrm{~mm}$ and a height of $40 \mathrm{~mm}$, inside the standard niobium resistance furnace of the FRM II. Liquid ${ }^{\text {nat }} \mathrm{Ge}$-samples were measured in full cylinder geometry with a diameter of $9 \mathrm{~mm}$ and a height of $40 \mathrm{~mm}$. The isotope mixture was measured in hollow cylindrical geometry with a diameter of $22 \mathrm{~mm}$ and $1.2 \mathrm{~mm}$ of sample thickness. In the quasielastic region the scattering signal from the empty container is more than an order of magnitude smaller than the scattering signal obtained for container and sample. Moreover, the inelastic signal is well separated from the quasielastic signal and thus dominates the time-of-flight signal below about 1 millisecond per meter. Above $1 \mathrm{~ms} \mathrm{~m}^{-1}$ the quasielastic scattering from the liquid sample centered around approximately $1.8 \mathrm{~ms} \mathrm{~m}^{-1}$ dominates the signal even in the case of ${ }^{\text {nat }} \mathrm{Ge}$ with its 7 times lower incoherent scattering cross section compared with the ${ }^{\text {nat } / 73} \mathrm{Ge}$ isotope mixture.

Measured time-of-flight spectra were normalized to a vanadium standard, corrected for self absorption and empty container scattering, and interpolated to constant $q$ in order to obtain $S(q, \omega)$.

In Fig. 1 , the measured $S(q, \omega)$ for ${ }^{\text {nat }} \mathrm{Ge}$ and ${ }^{\text {nat } / 73} \mathrm{Ge}$ are depicted for two different $q$-values of $0.4 \AA^{-1}$ and $0.6 \AA^{-1}$. All spectra are well described by a Lorentzian function:

$$
F(q, \omega)=\frac{A}{\pi} \frac{\Gamma_{1 / 2}(q)}{(\hbar \omega)^{2}+\left(\Gamma_{1 / 2}(q)\right)^{2}} \otimes R(q, \omega)+b(q)
$$

convolved with the energy resolution function $R(q, \omega)$ of the spectrometer. Here, $b(q)$ accounts for energy independent contributions to the signal and $\Gamma_{1 / 2}(q)$ is the half width at half maximum (HWHM) of the Lorentzian curve.

Figure 2a displays calculated Rayleigh- (dotted) and Brillouin (dashed) lines of a coherent dynamic structure factor for liquid $\mathrm{Ge}$ at $q=0.4 \AA^{-1}$. For the Brillouin lines, reported literature values of $c_{\mathrm{s}}=2691 \mathrm{~m} / \mathrm{s}$ [38] and 

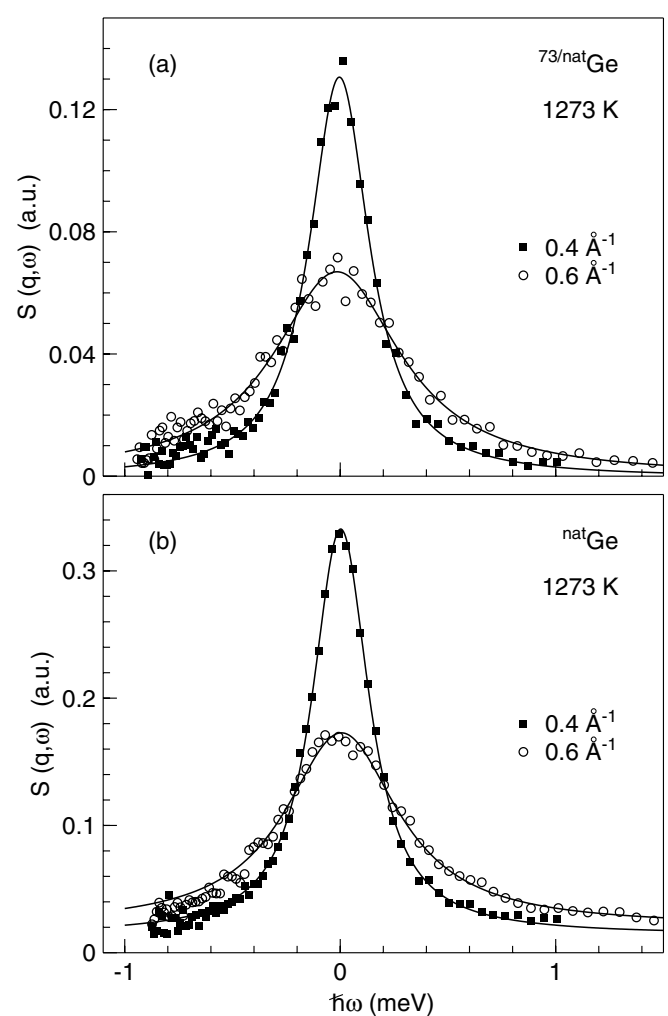

Figure 1. Dynamic structure factor $S(q, \omega)$ of (a) liquid ${ }^{\text {nat } / 73} \mathrm{Ge}$ and (b) liquid ${ }^{n a t} \mathrm{Ge}$ at wavenumbers of $0.4 \AA^{-1}$ and $0.6 \AA^{-1}$. Lines are fits with a Lorentzian function convolved with the energy resolution function (Eq. (3)). QENS spectra of liquid ${ }^{\text {nat } / 73} \mathrm{Ge}$ and ${ }^{\text {nat }} \mathrm{Ge}$ exhibit a comparable half width at half maximum.

$\Gamma_{\mathrm{s}} \simeq 2 \mathrm{meV}$ [34] were used. The thermal diffusivity $D_{\mathrm{T}}$ was chosen such that it matches the Rayleigh line of the X-ray structure factor reported in Ref. [34]. The half width at half maximum of approximately $4 \mathrm{meV}$ at $q=0.6 \AA^{-1}$ corresponds to a thermal diffusivity of $1.3 \cdot 10^{-7} \mathrm{~m}^{2} / \mathrm{s}$, in agreement with a rough estimate in Ref. [35].

It should be noted, that the thermal diffusivity may also be calculated according to $D_{\mathrm{T}}=\lambda_{\mathrm{T}} /\left(\rho c_{\mathrm{P}}\right)$. With literature values for the heat conductivity $\lambda_{\mathrm{T}}$ [39], the specific heat $c_{\mathrm{P}}[40]$ and the macroscopic density $\rho$ [41], a value of $D_{\mathrm{T}} \approx 2 \cdot 10^{-5} \mathrm{~m}^{2} / s$ is derived for a temperature of $T=1273 \mathrm{~K}$. This value is more than two orders of magnitude larger than the thermal diffusivity derived from the Rayleigh line in the X-ray structure factor [34]. Liquid Ge exhibits values for thermal and electrical conductivity that compare well to other liquid metals. In liquid metals and, in the absence of convective flow effects, transport of heat is governed by electronic contributions, which in turn do not contribute to the X-ray and neutron scattering signal, respectively. Accordingly, for the calculation of the spectra displayed in Fig. 2 we use the value of 1.3 . $10^{-7} \mathrm{~m}^{2} \mathrm{~s}^{-1}$ for $D_{\mathrm{T}}$ derived from inelastic X-ray scattering.

In Fig. $2 b$ a dynamic structure factor of liquid ${ }^{\text {nat } / 73} \mathrm{Ge}$ as seen by QENS is also depicted. Compared to the measured quasielastic line width the Rayleigh line appears as a flat background (accounted for by $b(q)$ in Eq. (3)). Brillouin lines lie outside the relevant energy range for
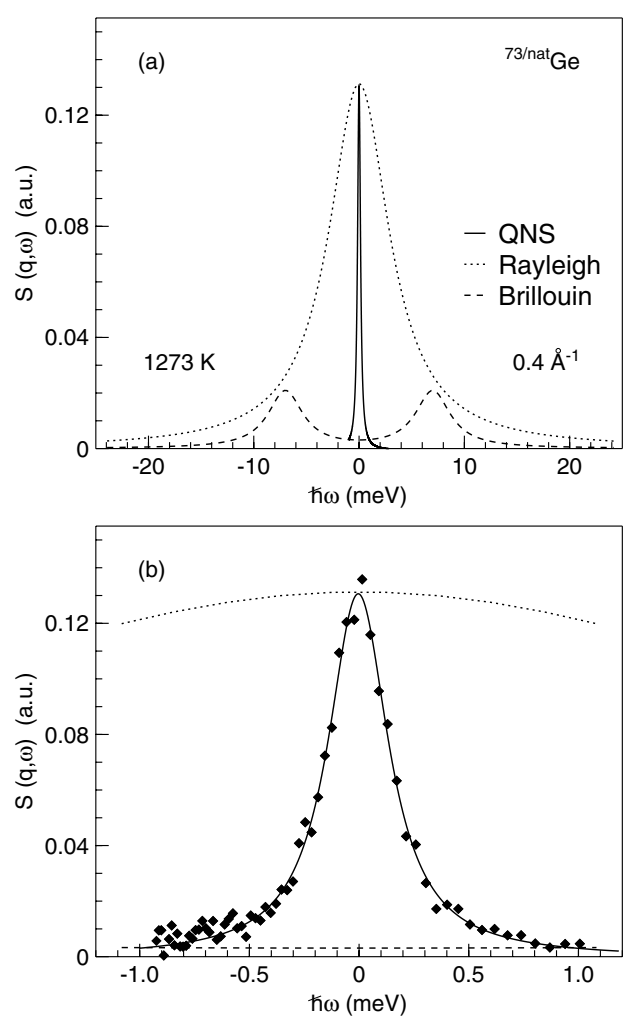

Figure 2. (a) Rayleigh- (dotted) and Brillouin (dashed) lines of the coherent dynamic structure for liquid Ge calculated using Eq. (1) (solid line) and QENS data on liquid ${ }^{\text {nat } / 33}$ Ge from Fig. 1 (diamonds). The peak height of the Rayleigh line is normalized to the peak height in the measured $S(q, \omega)$. The ratio of peak heights in Rayleigh and Brillouin lines was chosen according to Ref. [34]. (b) QENS spectrum with lines from (a). The half width at half maximum $\Gamma$ of the measured quasielastic line is below $0.2 \mathrm{meV}$. For the measured wavenumber range of $0.3<q<$ $0.8 \AA^{-1}$, Brillouin lines lie outside the relevant $\hbar \omega$-range and the Rayleigh line appears as an almost constant background in $\hbar \omega$.

our QENS spectra. The same picture emerges for the QENS spectra of nat $\mathrm{Ge}$ which show a comparable HWHM of their quasielastic line. Moreover, at a wavenumber of $0.6 \AA^{-1}$ the HWHM of the QENS spectra is about $0.3 \mathrm{meV}$, whereas the Rayleigh line exhibits a $\Gamma_{1 / 2}$ of about $4 \mathrm{meV}$. As a consequence, in the quasielastic spectra coherent scattering is well accounted for by an energy independent contribution $b_{q}$, that increases with increasing coherent scattering cross section (as seen in Fig. 1). However, in the intermediate $q$ range investigated here, the quasielastic Lorentzian line originates from incoherent scattering.

As demonstrated in Fig. 1 at the same value of $q$ spectra of nat/73 $\mathrm{Ge}$ and ${ }^{\text {nat }} \mathrm{Ge}$ exhibit a similar HWHM regardless of the large difference in their ratio of incoherent to coherent neutron scattering cross sections. This shows that at small $q$ self-diffusion coefficients can accurately be derived from QENS signals of liquid metals, even if the ratio of incoherent to coherent cross sections is small.

\section{Self-diffusion coefficients}

In order to derive the self-diffusion coefficient from QENS one has to assume that concepts developed in 


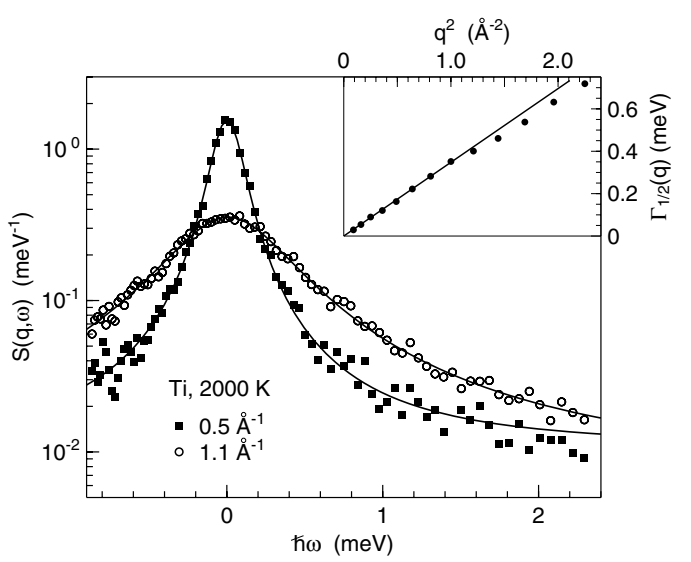

Figure 3. QENS spectra of liquid Titanium at $2000 \mathrm{~K}$ : Scattering law $S(q, \omega)$ at 2 different $q$ values. Lines are fits with Eq. (3). Insert: Half width half maximum of the quasielastic line $\Gamma_{1 / 2}(q)$ versus $q^{2}$. The line is a fit with Eq. (4).

the framework of hydrodynamics [1], i.e. in the limit of small- $q$ values, are valid also at $q$ values accessible by QENS, where, the $q$ range is usually restricted to values well above $0.1 \AA^{-1}$. With a structure factor maximum in liquid metals and alloys usually below $3 \AA^{-1}-$ at $q$ values certainly above $2 \AA^{-1}$ - coherent scattering contributions and deviations from hydrodynamics become increasingly important. This leaves a fairly limited $q$ range, where one can test - by a $q^{2}$ dependence of the quasielastic line width - whether hydrodynamics can be applied and thus selfdiffusion coefficients can be derived.

In the following we present results of a QENS experiment on liquid titanium at $2000 \mathrm{~K}$ [29]. In order to investigate up to which $q$ values hydrodynamic predictions can be utilized for the analysis of the neutron scattering spectra, molecular dynamics (MD) computer simulations were performed using using an embedded atom model for $\mathrm{Ti}$ [13]. The results show that in liquid Ti long range atomic transport can be measured up to about $1.2 \AA^{-1}$ with QENS.

QENS was performed at the neutron time-offlight spectrometer ToFToF of the FRM II using our electromagnetic levitation device, especially designed for neutron spectroscopy [26]. A roughly spherical, electrically conductive Ti sample with a mass of $1.5 \mathrm{~g}$ (about $10 \mathrm{~mm}$ in diameter), was levitated within an inhomogeneous electromagnetic radio frequency field. As a result of the radio frequency field, eddy currents are induced in the specimen. This leads to an inductive heating of the sample which allows melting of the specimen and to a force into the direction of low magnetic field strength such that gravity is compensated. The convective stirring induced by the inductive currents in combination with a large heat conductivity of the sample results in a homogeneous sample temperature that is measured contact-free with a two-color pyrometer. The absolute temperature is derived by calibrating the measured melting temperature to the literature value for $T_{\mathrm{m}}(\mathrm{Ti})$ of $1941 \mathrm{~K}$. The precision is about $\pm 5 \mathrm{~K}$. During the measurement the sample chamber was filled with 500 mbar Argon with $99.9999 \%$ purity. The data acquisition time was $100 \mathrm{~min}$ for the sample at $2000 \mathrm{~K}$.
We note that we recently developed an electrostatic levitation device (ESL) for application on neutron scattering as well as X-ray and neutron diffraction beamlines [27]. In ESL samples are containerlessly processed under high vacuum conditions $\left(<1 \times 10^{-6}\right.$ mbar $)$. A stable levitation of the liquid droplet is achieved by electrostatic forces adjusted with an active sample position control mechanism. An infrared laser beam $(\lambda=808 \mathrm{~nm})$ focused on the sample surface is used for heating and melting of the sample. One great advantage of ESL as compared to EML is the full sight of the neutron beam on the sample. With distances of the electrode system as large as $15 \mathrm{~mm}$ and a typical sample size of 5-6 mm scattering of the sample environment is negligible.

For the liquid $\mathrm{Ti}$ experiment a wavelength of the incident neutrons of $5.4 \AA$ was chosen giving access to a wavenumber $q$ ranging from $0.3 \AA^{-1}$ to $2.2 \AA^{-1}$ at zero energy transfer. The instrumental energy resolution function $R(q, \omega)$, measured at $300 \mathrm{~K}$ using a vanadium standard of similar size, can be well approximated by a Gaussian function with a half width at half maximum of $\simeq 40 \mu \mathrm{eV}$.

For titanium $\sigma_{\text {inc }}=2.87$ barn and $\sigma_{\text {coh }}=4.35$ barn. Well below the first structure factor maximum at $\simeq 2.65 \AA^{-1}[42,43]$ the signal is dominated by incoherent contributions and thus $S(q, \omega)$ describes the self motion of the atoms. As expected for the hydrodynamic regime [1], at small $q$, the QENS signal is well described by the Lorentzian function (Eq. (3)) and $\Gamma_{1 / 2}(q)$ follows a $q^{2}$ dependence at small $q$ (insert of Fig. 3). Above about $1.2 \AA^{-1}$ small but systematic deviations from the $q^{2}$ dependence emerge. For $q$ values $<1.2 \AA^{-1}$ the self diffusion coefficient $D$ can be calculated through $\Gamma_{1 / 2}(q)$ of the quasi-elastic line via:

$$
D=\frac{\Gamma_{1 / 2}(q)}{\hbar q^{2}}
$$

The resulting value of $D_{\mathrm{Ti}}=(5.3 \pm 0.2) \times 10^{-9} \mathrm{~m}^{2} \mathrm{~s}^{-1}$ is equal within error bars to the value of liquid Nickel $D_{\mathrm{Ni}}=$ $(5.0 \pm 0.3) \times 10^{-9} \mathrm{~m}^{2} \mathrm{~s}^{-1}$ at $2000 \mathrm{~K}$ [26], which will be addressed in the following section.

In MD simulations the self-diffusion coefficient can also be computed from the long-time limit of the mean square displacement $r^{2}(t)$ via the Einstein relation:

$$
D=\lim _{t \rightarrow \infty} \frac{\left\langle r^{2}(t)\right\rangle}{6 t} .
$$

The MD self-diffusion coefficient of liquid Ti obtained via the analysis of $S(q, \omega)$ with Eq. (3) and Eq. (4) are accurately described with the resulting self-diffusion coefficient of Eq. (5) up to $q$ values of about $1.2 \AA^{-1}$ [13]. In agreement with the experimental data above about $q=1.2 \AA^{-1}$ deviations from the hydrodynamic behavior come into play.

This emphasizes that at values of $q$ as large as about half the value of the first structure factor maximum, incoherent contributions dominate the quasielastic line shape and self-diffusion coefficients can accurately be derived on the atomic length scales that are accessible in QENS experiments. 


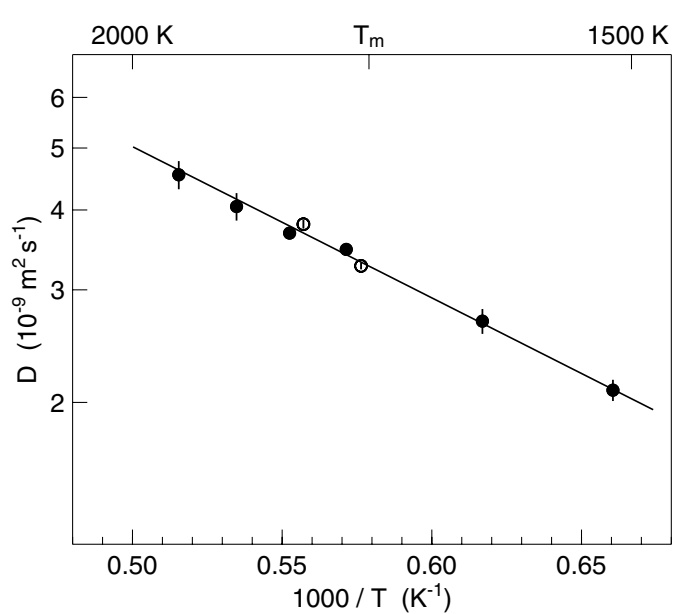

Figure 4. Ni self-diffusion coefficients $D$ as a function of inverse temperature in a range spreading from more than $200 \mathrm{~K}$ above to more than $200 \mathrm{~K}$ below the melting point. The full circles are the data points determined by the levitation experiment - the open circles are data points from the crucible experiment. The line is a fit with Eq. (6).

\section{Multiple scattering on levitated samples}

In order to address the impact of multiple scattering on the HWHM of the QENS signal, results from two experiments on liquid nickel are compared. $\mathrm{Ni}$ in natural isotope abundance is a strong incoherent scatterer with a cross section of $\sigma_{i}=5.2 \mathrm{barn}$. One experiment was performed in a special $\mathrm{Al}_{2} \mathrm{O}_{3}$ crucible with a sample geometry of a hollow cylinder, $0.6 \mathrm{~mm}$ wall thickness, a diameter of $22 \mathrm{~mm}$, and a sample height of $40 \mathrm{~mm}$ [30]. The other experiment was carried out on a liquid droplet of $8 \mathrm{~mm}$ in diameter using the EML apparatus described above [26]. The levitation experiment was performed on ToFToF with a similar set-up as described for liquid Ti. The crucible experiment was performed on the time-offlight spectrometer IN6 at the Institute Laue-Langevin with an incoming neutron wavelength of $5.1 \AA$ in a resistance furnace under vacuum. The levitation experiment was performed in a $350 \mathrm{mbar} \mathrm{He} / 4 \% \mathrm{H}_{2}$ gas atmosphere. The temperature of the melt was controlled via the flow of ultra pure cooling gas $\left(\mathrm{He} / 4 \% \mathrm{H}_{2}\right)$ which is injected by a nozzle that is installed below the sample. The addition of hydrogen gas is used to reduce oxide impurities on the sample surface enabling deeper undercoolings of the sample. Data acquisition times were three hours for temperatures below the melting point $\mathrm{T}_{m}=1726 \mathrm{~K}$ ), and one hour for temperatures above the melting point. The IN6 spectra were collected for 2-4 hours.

Figure 4 shows the resulting $\mathrm{Ni}$ self-diffusion coefficients in an Arrhenius diagramm. While the QENS measurements in the crucible were performed in a narrow regime of temperatures above the melting point, the application of the electromagnetic levitation technique gave access to a significantly wider temperature range, including also the metastable regime of the undercooled liquid.

Over a large temperature range - from more than $200 \mathrm{~K}$ above to more than $200 \mathrm{~K}$ below the melting point of $1726 \mathrm{~K}$ - the temperature dependence of the Ni selfdiffusion coefficient can be described by an Arrhenius law,

$$
D=D_{0} \exp \left(-E_{A} / k_{B} T\right),
$$

with an activation energy $E_{A}=470 \pm 30 \mathrm{meV}$ per atom and a pre-factor $D_{0}=77 \pm 8 \times 10^{-9} \mathrm{~m}^{2} \mathrm{~s}^{-1}$. This also shows that the change from a liquid in thermodynamic equilibrium to a metastable liquid at temperatures below the melting point is not reflected in its atomic dynamics.

Figure 4 also shows that the values of the self-diffusion coefficients from the two experiments are equal within error bars, that are typically in the range of a few percent. Apparently, the much thicker sample geometry in the levitation experiment does not hamper the determination of the Ni self-diffusion coefficients. It should be noted, that for most other metals the incoherent cross section is much smaller than that for $\mathrm{Ni}$ and, therefore, our data show that effects of multiple scattering on the values of the self-diffusion coefficients in liquid metals from QENS experiments are negligible. Multiple scattering effects on the QENS signal at small $q$ involving coherent QENS can only become important if the $q$ range around the first structure factor maximum is within the dynamic range of the measurement. With a wavelength of the incoming neutrons of $5.1 \AA$ and above, this was not the case in the experiments presented here.

\section{Temperature dependence of self-diffusion}

Due to the difficulties arising in classical LC diffusion experiments discussed in the introduction, there are very few reliable data sets on the temperature dependence of the self diffusion in liquid metals. Recently, we have also measured copper self-diffusion coefficients with QENS in a broad temperature range above the melting point [19]. Liquid copper has emerged as a simple model system in computer simulations to study atomic transport properties, nucleation, melting, and crystal growth, as well as relations of melt structure and self diffusion. In such studies experimental atomic diffusion coefficients serve as a benchmark for the quality of simulations with effective model potentials as well as for ab initio simulations.

The setup on ToFToF with a wavelength of the incident neutrons of $\lambda=7 \AA$ gives an accessible $q$ range of about 0.4 to $1.6 \AA^{-1}$ at zero energy transfer at an instrumental energy resolution of about $25 \mu \mathrm{eV}$ (HWHM). Copper has an incoherent scattering cross section of 0.55 barn, and at the melting point, a structure factor maximum of $\simeq 2.9 \AA^{-1}$. The measurement was carried out in a resistance furnace under vacuum using a thin-walled $\mathrm{Al}_{2} \mathrm{O}_{3}$ container that provides a hollow cylindrical sample geometry with $22 \mathrm{~mm}$ in diameter, $40 \mathrm{~mm}$ in height and a $2 \mathrm{~mm}$ wall thickness. Data were collected at temperatures above the melting point of $\mathrm{Cu}$ at $1358 \mathrm{~K}$ in a range from $1370 \mathrm{~K}$ to $1620 \mathrm{~K}$ in steps of $50 \mathrm{~K}$ for 90 minutes each, and are shown in Fig. 5. A best fit with Eq. (6) results in $E_{\mathrm{A}}=(337 \pm 5) \mathrm{meV}$ per atom and a $D_{0}=(58.7 \pm 3) \times 10^{-9} \mathrm{~m}^{2} \mathrm{~s}^{-1}$. Notably, at the respective melting temperature the self-diffusion coefficients of $\mathrm{Cu}$ 


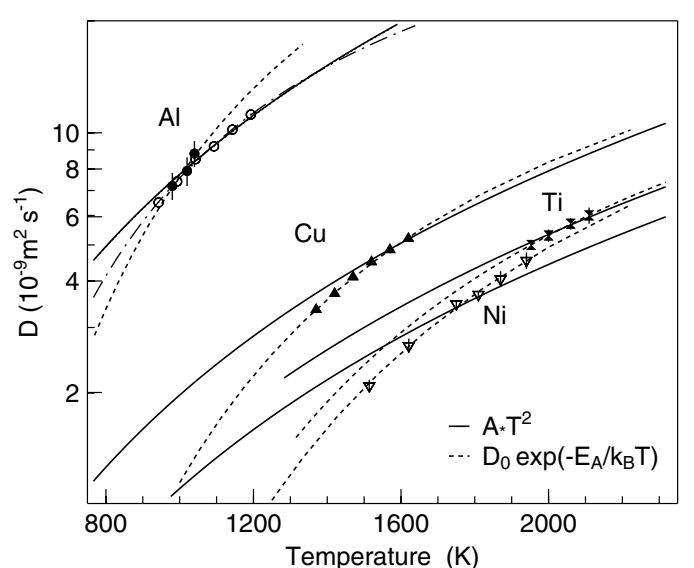

Figure 5. Self-diffusion coefficients for various liquid metals that were recently studied by QENS. Dashed lines represent fits with an Arrhenius-type temperature dependence (Eq. (6)). Solid lines the best fit with a $T^{2}$ dependence. Whereas the Arrhenius-fits give an excellent description of all data sets, a $T^{2}$ dependence is not in line with our data. In the case of liquid $\mathrm{Al}$, solid circles are data from incoherent QENS and open circles are derived from coherent QENS (Ref. [32]). The dashed-dotted line is a $T^{2}$ fit to the incoherent data - the solid line to the coherent data..

$\left(3.27(4) \times 10^{-9} \mathrm{~m}^{2} \mathrm{~s}^{-1}\right)$ and $\mathrm{Ni}\left(3.28(7) \times 10^{-9} \mathrm{~m}^{2} \mathrm{~s}^{-1}\right)$ are equal within error bars.

In contrast to the LC data set by Henderson and Yang [44] used as the text book reference for simulations, our QENS data are significantly below the reported values. In the latter, convective effects cause a relatively large scatter and the resulting activation energy is about $25 \%$ larger than for the QENS results [19]. Diffusion coefficients reported in classical and ab-initio MD simulations on liquid $\mathrm{Cu}$ exhibit a scatter of about $\pm 50 \%$ around our experimental values. This shows that, while MD simulations (classical or ab-initio) can be used as a tool to reveal microscopic mechanisms, it is in general not applicable for the determination of absolute values of materials properties. On the other hand, accurate values of liquid diffusion coefficients from experiments can serve as a benchmark for the improvement of model potentials as has been successfully demonstrated for the case of liquid titanium [13].

Liquid aluminum is a very challenging system for the study of self diffusion with QENS due to its very small incoherent scattering cross section of only 0.008 barn. In an experiment on ToFToF a solid Al-cylinder of $21 \mathrm{~mm}$ in diameter and $40 \mathrm{~mm}$ in height consisting of high purity $\mathrm{Al}$ (99.9999\%, Hydro-Aluminium) was used. The sample was melted in a thin-walled $\mathrm{Al}_{2} \mathrm{O}_{3}$ hollow cylinder of $23 \mathrm{~mm}$ outer diameter at $0.5 \mathrm{~mm}$ wall thickness in a standard niobium resistance furnace. The QENS experiments were performed on ToFToF using incoming neutron wavelengths of $7.0 \AA$ and $10 \AA$ respectively. Results obtained at these two wavelengths compare well with each other. In Ref. [31], it is shown that even in this unfavorable case of liquid Aluminum reliable selfdiffusion coefficients can be obtained. Data are depicted in Fig. 5.

Compared with liquid $\mathrm{Cu}, \mathrm{Ti}$, and $\mathrm{Ni}$, self-diffusion in liquid $\mathrm{Al}$ is significantly faster. Therefore, the resulting
HWHM of the QENS signal is significantely larger. Together with the small incoherent scattering cross section, it becomes increasingly difficult with increasing temperature, and hence, increasing line width to resolve the quasielastic line. In order to extend the data to larger temperatures, our self-diffusion coefficients from incoherent scattering are compared to data from a coherent scattering experiment at $q$ values around the structure factor maximum at $q_{0} \simeq 2.6 \AA^{-1}$ [32]. There diffusion coefficients were obtained using a modified Enskog hardsphere model. Both data sets agree within error bars. This shows that for liquid Al the model assumptions are reasonable.

As has been recently shown in a QENS experiment on a viscous $\mathrm{Zr}$-Ti-Ni-Cu-Be alloy the corresponding line width from the incoherent signal at small $q$ and from the coherent signal in the structure factor maximum exhibit the same temperature dependence [45]. If this would hold in general, the temperature dependence of self-diffusion coefficient can also be derived in systems with a small incoherent, but a reasonable coherent scattering cross section. Thus an Ansatz to calibrate a set of coherent QENS data, spanning a large temperature range, with one self-diffusion coefficient measured with incoherent QENS at low temperature, or in a LC tracer diffusion experiment under microgravity conditions and in-situ monitored with X-ray radiography, appears to be promising.

Figure 5 shows the self-diffusion coefficients that we have recently measured with QENS. All data sets are best described with an Arrhenius-type temperature behavior. This is in contrast to the $T^{2}$ behavior reported for metallic melts with lower density of packing such as $\mathrm{Sn}, \mathrm{Pb}, \mathrm{In}$ and $\mathrm{Sb}[46,47]$. There, LC experiments were performed in space under microgravity conditions, where the absence of buoyancy driven convective flow resulted in the required precision of the diffusion coefficients. As shown in Fig. 5 our data sets, especially the ones that span a large temperature range, can not be described with $D(T) \propto$ $T^{2}$. As a consequence, a $T^{2}$ behavior of the temperature dependence of the self-diffusion coefficient does certainly not hold as a general rule in single-component liquid metals around the melting point.

\section{Conclusions}

To summarize, it has been shown in this review, that quasielastic neutron scattering is a versatile tool for the study of self-diffusion in liquid metals. Even in systems with a small incoherent scattering cross section, accurate diffusion data can be measured with reasonable error bars. With increasing incoherent cross section, errors originating from statistics and data reduction are within a few percent of the absolute value. The accuracy of the experimental result is then limited by technical restrictions, e.g. the measurement of the absolute value of the sample temperature. Finally, it has to be emphasized that the conclusions reported here hold for fairly-dense and singlecomponent liquid metals. In multicomponent systems, coherent cross terms may contribute to the QENS signal in the $q$ and energy range addressed here. Therefore, the measurement of self-diffusion coefficients in alloys is only feasible if incoherent scattering of one alloy component 
dominates the QENS signal, e.g. in Al-Ni [48] or Zr-Ni [49] alloys.

It is a pleasure to thank all my colleagues that have contributed to the research reported here for a fruitful and long-standing collaboration, the Institut Laue-Langevin (ILL) and the HeinzMaier-Leibnitz neutron source (FRM-2) for beamtime, Florian Kargl for the preparation of Fig. 5, and the latter, Zach Evenson, and Thomas Voigtmann for a critical reading of the manuscript.

\section{References}

[1] J. P. Boon and S. Yip, Molecular Hydrodynamics, (McGraw-Hill, New York, 1980)

[2] T. Iida and R.I.L. Guthrie, The Physical Properties of Liquid Metals (Clarendon Press, Oxford, 1993)

[3] E. L. Cussler, Diffusion - Mass Transfer in Fluid Systems (University Press, Cambridge, 2009)

[4] U. Balucani, M. Zoppi, Dynamics of the Liquid State, (Clarendon, Oxford, 1994)

[5] J. Brillo, A. I. Pommrich, A. Meyer, Phys. Rev. Lett. 107, 165902 (2011)

[6] M. Rappaz and W. J. Böttinger, Acta Mater. 47, 3205 (1999)

[7] W. J. Böttinger, J. A. Warren, C. Beckermann and A. Karma, Annu. Rev. Mater. Res. 32, 163 (2002)

[8] R. Heringer, C.-A. Gandin, G. Lesoult and H. Henein, Acta Mater. 54, 4427 (2006)

[9] K. Binder and W. Kob, Glassy Materials and Disordered Solids: An Introduction to their Statistical Mechanics, (World Scientific, Singapore 2005)

[10] W. Götze, in Liquids, Freezing and the Glass Trasition, Proceedings of the Les Houches Summer School of theoretical Physics, Session LI, 1989, edited by J.-P. Hansen, D. Levesque, and J. ZinnJustin (North-Holland, Amsterdam, 1991) 287

[11] D. M. Herlach, P. Galenko, and D. Holland-Moritz, Metastable solids from undercooled melts, edited by R.W. Cahn (Pergamon Materials Series, Elsevier, 2007)

[12] G. Kasperovich, A. Meyer, and L. Ratke, Int. Foundry Res. 62, 8 (2010)

[13] J. Horbach, R. Rozas, T. Unruh, A. Meyer, Phys. Rev. B 80, 212203 (2009)

[14] P. Kuhn, J. Horbach, F. Kargl, A. Meyer, and T. Voigtmann, Phys. Rev. B 90, 024309 (2014)

[15] A. Griesche, M.-P. Macht, J.-P. Garandet, and G. Frohberg, J. Non Cryst. Solids 336, 173 (2004)

[16] T. Masaki, T. Fukazawa, S. Matsumoto, T. Itami, and S. Yoda, Meas. Sci. Technol. 16, 327 (2005)

[17] V. Zöllmer, K. Rätzke, F. Faupel, and A. Meyer, Phys. Rev. Lett. 90, 195502 (2003)

[18] A. Meyer, New Experiments on Diffusion of Mass in Liquid Metals, High Temp. High Press. 42 (2013)

[19] A. Meyer, Phys. Rev. B 81, 012102 (2010)

[20] B. Zhang, A. Griesche, and A. Meyer, Phys. Rev. Lett. 104, 035902 (2010)

[21] F. Kargl, M. Engelhardt, F. Yang, H. Weis, P. Schmakat, B. Schillinger, A. Griesche, and A. Meyer, J. Phys.: Condens Matter 23, 254201 (2011)

[22] F. Kargl, E. Sondermann, H. Weis, and A. Meyer, High Temp. High Press. 42, 1 (2013)
[23] H. J. V. Tyrrell, and K. R. Harris, Diffusion in Liquids, (Butterworths, London, 1984)

[24] C. Morkel, and W. Gläser, Phys. Rev. A 33, 3383 (1986)

[25] A. Meyer, Phys. Rev. B 66, 134205 (2002)

[26] A. Meyer, S. Stüber, D. Holland-Moritz, O. Heinen, and T. Unruh, Phys. Rev. B 77, 092201 (2008)

[27] T. Kordel, D. Holland-Moritz, F. Yang, J. Peters, T. Unruh, T. Hansen, and A. Meyer, Phys. Rev. B 83, 104205 (2011)

[28] H. Weis, T. Unruh, and A. Meyer, High Temp. High Press. 42, 39 (2013)

[29] A. Meyer, J. Horbach, O. Heinen, D. Holland-Moritz, and T. Unruh, Defect Diff. Forum 289-292, 609 (2009)

[30] S. Mavila Chathoth, A. Meyer, H. Schober, and F. Juranyi, Appl. Phys. Lett. 85, 4881 (2004)

[31] F. Kargl, H. Weis, T. Unruh, and A. Meyer, J. Phys.: Conf. Ser. 340, 012077 (2012)

[32] F. Demmel, D. Szubrin, W.-C. Pilgrim, and C. Morkel, Phys. Rev. B 84, 014307 (2011)

[33] J.P. Hansen, I.R. McDonald, Theory of Simple Liquids London: Academic Press Inc. (1986)

[34] S. Hosokawa, Y. Kawakita, W.-C. Pilgrim, and H. Sinn, Phys. Rev. B 63, 134205 (2000)

[35] J.D. Chai, D. Stroud, J. Hafner, and G. Kresse, Phys. Rev. B 67, 104205 (2003)

[36] T. Unruh, A. Meyer, J. Neuhaus and W. Petry, Neutron News 1, 23 (2007)

[37] T. Unruh, J. Neuhaus and W. Petry, Nucl. Instr. Meth. A 580, 1414 (2007)

[38] M. Hayashi, H. Yamada, N. Nabeshima, and K. Nagata, International Journal of Thermophysics 28, 83 (2007)

[39] E. Yamasue, M. Susa, H. Fukuyama, and K.J. Nagata, J. of Cryst. Growth 234, 121 (2002)

[40] Q. Li, Y.Y. Zhu, R.P. Liu, G. Li, M.Z. Ma, J.K. Yu, J.L. He, Y.J. Tian, and W.K. Wang, Appl. Phys. Lett. 85, 558 (2004)

[41] S.M. Chathoth, B. Damaschke, K. Samwer, and S. Schneider, Appl. Phys. Lett. 93, 071902 (2008)

[42] G. W. Lee, A. K. Gangopadhyay, K. F. Kelton, R. W. Hyers, T. J. Rathz, J. R. Rogers, and D. S. Robinson, Phys. Rev. Lett. 93, 037802 (2004)

[43] D. Holland-Moritz, O. Heinen, R. Bellissent, and T. Schenk, Mat. Sci. Eng. A 449, 42 (2007)

[44] J. Henderson and L. Yang, AIME Trans. 221, 72 (1961)

[45] F. Yang, T. Unruh, and A. Meyer, EPL 107, 26001 (2014)

[46] G. Mathiak, A. Griesche, K.H. Kraatz, and G. Frohberg, J. Non-Cryst. Sol. 205-207, 412 (1996)

[47] T. Itami, T. Masaki, H. Aoki, S. Munejiri, M. Uchida, S. Masumoto, K. Kamiyama, and K. Hoshino, J. Non-Cryst. Sol. 312-314, 177 (2002)

[48] S. K. Das, J. Horbach, M. M. Koza, S. Mavila Chathoth, and A. Meyer, Appl. Phys. Lett. 86, 011918 (2005)

[49] T. Voigtmann, A. Meyer, D. Holland-Moritz, S. Stüber, T. Hansen, and T. Unruh, EPL 82, 66001 (2008) 